This item was submitted to Loughborough's Research Repository by the author.

Items in Figshare are protected by copyright, with all rights reserved, unless otherwise indicated.

\title{
Vertical flotation of particles in a paramagnetic fluid
}

\section{PLEASE CITE THE PUBLISHED VERSION}

https://doi.org/10.1016/j.powtec.2014.03.067

\section{PUBLISHER}

(c) Elsevier

\section{VERSION}

NA (Not Applicable or Unknown)

\section{PUBLISHER STATEMENT}

This work is made available according to the conditions of the Creative Commons Attribution-NonCommercialNoDerivatives 4.0 International (CC BY-NC-ND 4.0) licence. Full details of this licence are available at: https://creativecommons.org/licenses/by-nc-nd/4.0/

\section{LICENCE}

CC BY-NC-ND 4.0

\section{REPOSITORY RECORD}

Liu, Shi-Xiao, Mark C. Leaper, and Nicholas J. Miles. 2017. "Vertical Flotation of Particles in a Paramagnetic Fluid". figshare. https://hdl.handle.net/2134/26842. 


\title{
Vertical Flotation of particles in a paramagnetic fluid
}

\author{
S. Liu ${ }^{1 *}$, M. Leaper ${ }^{2}$, N.J. Miles ${ }^{3}$
}

1. Process and Environmental Research Division, Faculty of Engineering, University of Nottingham, University Park, Nottingham, NG7 2RD, United Kingdom

2. Chemical Engineering and Applied Chemistry, Aston University, Birmingham B4 7ET

3. Faculty of Science and Engineering, University of Nottingham Ningbo China, Taikang East Road, Ningbo, 315100, China

\section{Abstract}

Hirota et al. (2004) found that the magneto-Archimedes force could be used to levitate biological materials at different heights in pressurized oxygen, providing the possibility to separate them. However the magnetic levitation of mineral particles has not been widely explored. With this in mind some preliminary experiments were performed by levitating pure mineral materials in a paramagnetic solution manganese (II) chloride. Besides the report of levitation heights of various mineral particles in manganese (II) chloride solution, the lines obtained from the basic formula provided by previous researchers were compared with experimental data. The act of cryogenic paramagnetic fluid in the magneto-Archimedes levitation was also demonstrated. The obtained results are compared with the same particle levitation heights in manganese (II) chloride solution.

Keywords: magneto-Archimedes; levitate; shape; cryogenic

\section{Introduction}

As early as 1991, a water droplet, bismuth metal, antimony, plastic, wood, alcohol and acetone have been levitated successfully in air as the separation medium [2]. Among the levitated materials, the levitation of water needed the strongest magnetic field strength [2]. Graphite is one of the best choices for levitation because it has a larger diamagnetic susceptibility than most other materials and has a relative low density. Therefore a piece of graphite at room temperature can be levitated using powerful permanent magnets $\left(\mathrm{Nd}_{2} \mathrm{Fe}_{14} \mathrm{~B}\right)$ 
in open air [3]. Copper has much higher density so it is very difficult to levitate in air even they were placed on as strong as the four blocks array permanent magnet [4]. When changing the magneto-Archimedes agent from air to dysprosium nitrate $\left(\mathrm{Dy}\left(\mathrm{NO}_{3}\right)_{3}\right)$, a piece of copper could be levitated effectively [5]. Also it was found out that carbon, $\mathrm{Si}$, and $\mathrm{Ti}$ were levitated at different positions in the separation medium 2.67 M Dy $\left(\mathrm{NO}_{3}\right)_{3}$ using a standard laboratory electromagnet with magnet field strength 1.4T [5]. Hirota et al in 2004 successfully used magneto-Archimedes separation to separate biological materials [6].

In the presence of a background fluid, magneto-Archimedes levitation applies the buoyancy principle to the levitation in a magnetic field, which could be modified as shown by the following formula [7]:

$\frac{\chi_{0}}{\mu_{0}} B \frac{d B}{d z}-\rho_{0} g-\frac{\chi_{f}}{\mu_{0}} B \frac{d B}{d z}+\rho_{f} g=0$

Where $\chi_{0}$ and $\rho_{0}$ are the magnetic susceptibility and density of the levitating object respectively, $\chi_{f}$ and $\rho_{f}$ are those of the background fluid. For the vertical direction magnet field gradient, the particle will endure four types of forces. Similar to wood in water, the particle in the media around it will receive a weight and buoyancy force provided by the media. Also the particle will be influenced by two further types of force, magnetic attraction and the magnetic levitation, which are related to the magnetic field strength. Here the levitation is known as the Magneto-Archimedes effect and the media called the Magneto-Archimedes agent [8-10].

Over the past decade a considerable amount of research has been conducted at the University of Nottingham on the levitation of particles under high magnetic fields and gradients [11-13]. This paper explores a new concept to separate particles with different density and magnetic susceptibility.

\section{Experimental procedure}

\subsection{Materials}

\subsubsection{The preparation and properties of magneto-Archimedes agent fluid}

The magneto-Archimedes solution used in this chapter is manganese (II) chloride solution. Different concentrations of manganese (II) chloride solution were prepared (ie, $2 \mathrm{M}, 3 \mathrm{M}$ and 4 
M) by dissolving the manganese (II) chloride crystal in distilled water to get a clear pink solution.

The mass susceptibility of the manganese (II) chloride solution at $2 \mathrm{M}, 3 \mathrm{M}, 4 \mathrm{M}$ can be obtained by calculation from Andres (1976) [14], which stated that the mass susceptibility of an aqueous solution of a paramagnetic salt could be obtained from the formula below[14]:

$x_{\text {total }}=C_{\text {salt }} x_{\text {salt }}+\left(1-C_{\text {salt }}\right) x_{\text {water }}$

$\boldsymbol{C}_{\text {salt }}=\frac{m_{\text {salt }}}{m_{\text {total }}}$

$k=\rho \chi * 4 \pi * 10^{-3}$

The corresponding volume magnetic susceptibility of $2 \mathrm{M}, 3 \mathrm{M}$ and $4 \mathrm{M}$ manganese (II) chloride solution were calculated and summarised in Table. 1.

\subsubsection{The preparation and properties of pure mineral samples}

In this research, various pure minerals and experimental materials were investigated. The minerals included pyrite, quartz, calcite, chalcopyrite, dolomite, galena, goethite, hematite, rutile, sphalerite and wolframite. Other materials were also used such as sand and glass. In Table. 2, the chemical form and mass magnetic susceptibilities were obtained from Gregory Bottley and Lloyd, [14] and [19]. The density were measured by Accupyc 1330 Helium pycnometer and the volume magnetic susceptibility were achieved by Formula (4). They were initially broken by a hammer, sieved to produce fine sized particles, and then dry screened to extract various size fractions for testing.

\subsection{Superconducting Magnet}

The experiments were performed using an Oxford Instruments Minimum Condensed Volume (MCV) superconducting magnet, which had a $5 \mathrm{~cm}$ diameter open bore with the maximum magnet central field being about 17 Tesla in the magnet bore, and the maximum $\mathrm{BdB} / \mathrm{dZ}$ field gradient about $\pm 1470 \mathrm{~T}^{2} \mathrm{~m}^{-1}$. The picture of the superconducting magnet is shown in Fig. 1a. The maximum field strength position is about $19 \mathrm{~cm}$ down into the bore from the top plate of the superconducting magnet. The magnet field strength plot is shown in Fig. 1b. 


\subsection{Design of Experimental Procedure}

\subsubsection{Classical magneto-Archimedes levitation}

To investigate vertical direction magnetic levitation, a small circular container was placed inside the superconducting magnet bore centre hole. The container was made of glass with an internal diameter of $23 \mathrm{~mm}$ and height of $85 \mathrm{~mm}$ and was filled with about $35 \mathrm{ml}$ of manganese (II) chloride solution. This container was set on a small plate about $2 \mathrm{~cm}$ below the top surface of the superconducting magnet as shown in Fig. 2 below.

\subsubsection{Comparison of the levitation positions of different pure mineral particles}

The levitation heights of the minerals in manganese (II) chloride solution were recorded using the same container for comparison under different magnetic field strengths. In addition the effect of solution density on levitation was assessed by adjusting the concentrations of the manganese (II) chloride solution.

\subsubsection{Experiments to confirm the magneto-Archimedes formula}

As mentioned, the levitation of objects should satisfy the condition in formula (5).

$$
V \rho_{P} g-V \rho g-\left(\frac{k_{p} V B B^{\prime}}{\mu_{0}}-\frac{k V B B^{\prime}}{\mu_{0}}\right)=0
$$

In this formula,

$$
B B^{\prime}=\frac{\left(\rho_{p}-\rho\right) g}{k_{p}-k} \mu_{0}
$$

As the materials which can be levitated in the fluid always have much lower magnetic volume susceptibility so the $k_{p}$ can be ignored in comparison to $k$. Then the formula (6) can be displayed as below.

$$
B B^{\prime}=\frac{\mu_{0} g}{k}\left(\rho-\rho_{p}\right)
$$

So if $B B^{\prime}$ is set as the $\mathrm{y}$-axis and $\rho_{p}$ as the $\mathrm{x}$-axis then the slope $\frac{\mu_{o} g}{k}$ and $\rho$ can be determined from Table. 1. The line to satisfy Formula (7) can be achieved based on a known 
magneto-Archimedes fluid such as $2 \mathrm{M}$ manganese (II) chloride solution. The experimental data of $B B^{\prime}$ to allow the particles with different densities levitated in $2 \mathrm{M}$ manganese (II) chloride solution were marked in the graphs and compared with the line obtained by the formula using 2M manganese (II) chloride solution as agent fluid.

\subsubsection{Effect of the shape of the container}

Based on the findings above that the particles were repulsed to the wall of the container, the shape of the container was changed to elliptical. The influence of the geometry on the vertical direction magnetic levitation experiment was recorded in Fig.13.

\subsubsection{The liquid oxygen and the mixture of liquid oxygen and nitrogen}

Because the oxygen molecule has two unpaired electrons in the anti-bonding $2 \mathrm{Mg}$ orbitals and they form a spin triplet so that the oxygen has quite strong paramagnetic property [11]. Liquid nitrogen does not have any paramagnetic property. So in this section, the act of $100 \%$ liquid oxygen and the mixture fluid as magneto-Archimedes agent were investigated at vertical direction magneto-levitation. The composition of the mixture fluid is mainly based on the idea about the composition of the air. So the composition of the mixture fluid was set to $25 \%$ oxygen and $75 \%$ nitrogen in volume which is quite close to the air composition (22\% oxygen and $78 \%$ nitrogen ).

\section{Results and Discussion}

\subsection{The classical magneto-Archimedes levitation}

Initial observations, with a single particle size fraction (ie, $212 \mu \mathrm{m}$ ), indicated that the particles formed a ring against the container wall as shown in Fig.3.

When two different mineral particle species were present, according to their different mass magnetic susceptibility and/or different densities, they will be floating at different height positions in the tube so that can be separated clearly. A photograph of this is shown in Fig.4.

Fig.4 shows a top ring of quartz particles and a lower ring of pyrite particles, which are clearly separated. It was considered that this could provide a basic method to separate mineral particles in the vertical direction. The results were consistent with the conclusions obtained by previous work $[2,20,7,6]$. The levitation positions of the particles remained static in relation 
to the top surface of the magnet even if the container was moved up or down. So the distance from the magnet top to the levitation position of the particles was fixed when the field strength was constant. The particles size range examined in this experiment was $212 \mu \mathrm{m}-1.2 \mathrm{~mm}$ and $53 \mu \mathrm{m}-106 \mu \mathrm{m}$ in diameter. It was found out that the levitation positions were also independent from the particle size mentioned above. It only depended on the field strength, particles and agent fluid magnetic susceptibility.

The reason why the particles were repulsed to the tube wall is that the manganese(II) chloride solution is paramagnetic and stand a force which attracted them to the magnet bore centre which is a hole in our superconducting magnet equipment.

The particles in the fluid faced a corresponding opposite force and be repulsed to the tube wall (Fig.16)

\subsection{Comparison of the levitation positions of different pure mineral particles}

The results of the levitation positions of the different particles at different field strengths are shown in Fig.5, Fig. 6 and Fig.7 below.

Because the platform which held the tube was about $2 \mathrm{~cm}$ below from the top of the magnet, the Y-axis, which states the height above the magnet, starts from $-1 \mathrm{~cm}$ to almost the height of the bottle which is about $8 \mathrm{~cm}$. As can be seen in Fig.5 the higher the field strength, the higher the particles levitate. Also the particles with different mass susceptibility levitated at different positions in the bottle. For the dolomite and calcite materials, as the magnetic field strength increased, the difference between the two materials levitation positions seems decreased. For the pyrite and rutile materials, as the magnetic field strength increased, the two materials become separated more effectively. The same condition happened to dolomite and sphalerite particles.

It is shown in the Fig.5 that the particles with similar density e.g quartz, calcite and dolomite, the levitation positions are the quartz particles occupy the highest position, calcite particles the second and dolomite particles the third. It follows that for particles with similar density, the magnetic susceptibility is the main factor to decide their levitation positions. These results are similar to results obtained by lkezoe et al. (2002) [7].

Fig.6 and Fig.7 are similar to the Fig.5 using different concentrations of manganese (II) chloride solution (3M and $2 \mathrm{M})$. They show as the concentration of the manganese (II) chloride 
solution decreased, the height of the particles float lower. And it was more difficult for the particles to be levitated at relatively lower $B_{c}$.

Fig.8 illustrates the positions in which the different minerals levitate in 4M manganese (II) chloride solution. Vertical direction magneto-Archimedes levitation can be applied to separate mineral particles. The densities of particles have bigger difference, the gaps of the levitation heights were bigger so that the separation is more effective.

From Formula (1) in this paper, we can see that the different mineral particles levitation positions depends on the combined effect of density and mass magnetic susceptibility (x). In Fig. 8 the particles with similar densities stay together e.g quartz particles (density $2644 \mathrm{~kg} / \mathrm{m}^{3}$ ) and calcite particles (density $2710 \mathrm{~kg} / \mathrm{m}^{3}$ ) or sphalerite particles (density $4000 \mathrm{~kg} / \mathrm{m}^{3}$ ) and rutile particles (density $4200 \mathrm{~kg} / \mathrm{m}^{3}$ ). Mineral particles whose density have the largest difference such as dolomite particles (density about $2974 \mathrm{~kg} / \mathrm{m}^{3}$ ) and sphalerite particles (density about $4000 \mathrm{~kg} / \mathrm{m}^{3}$ ) separate well and the levitation positions have the largest gap.

From Table 2 in this paper, the particles with similar magnetic mass susceptibility $(x)$ are quartz $\left(X_{\mathrm{p}}=-5.7 \times 10^{-9}\left(\mathrm{~m}^{3} \cdot \mathrm{kg}^{-1}\right)\right)$, calcite $\left(X_{\mathrm{p}}=-3.8 \times 10^{-9}\left(\mathrm{~m}^{3} \cdot \mathrm{kg}^{-1}\right)\right)$, galena $\left(X_{\mathrm{p}}=-4.4 \times 10^{-9}\right.$ $\left.\left(\mathrm{m}^{3} \cdot \mathrm{kg}^{-1}\right)\right)$ and sphalerite $\left(X_{\mathrm{p}}=-3.3 \times 10^{-9}\left(\mathrm{~m}^{3} \cdot \mathrm{kg}^{-1}\right)\right)$ particles. In Fig.8, quartz and calcite particles were levitated at similar positions, because these two mineral particles still have similar densities ( quartz density $2644 \mathrm{~kg} / \mathrm{m}^{3}$, calcite density $2710 \mathrm{~kg} / \mathrm{m}^{3}$ ). Galena, sphalerite particles were levitated at very different positions although they have similar magnetic mass susceptibility, because they still have different densities (galena density $7230 \mathrm{~kg} / \mathrm{m}^{3}$, sphalerite density $4000 \mathrm{~kg} / \mathrm{m}^{3}$ ).

The effect of particle density has bigger influence than the effect of particle magnetic mass susceptibility.

For the quartz particles, the gap between the levitation positions at $B_{c}=8$ and $9 T$ is the largest which can be seen from Fig.9. As the magnetic field strength increased, the gap between the levitation positions decreased. For the same particles the higher magnet bore centre field strength, the higher particle levitation position in the magneto-Archimedes agent manganese (II) chloride solution. 


\subsection{Experiments to confirm the magneto-Archimedes formula}

To achieve the magneto-Archimedes levitation, the Formula (6) should be satisfied. The slope of the formula $\frac{\mu_{o} g}{k}$ and the $y$-axis intercept $\frac{-\mu_{0} g \rho}{k}$ can be obtained based on the chosen fixed magneto-Archimedes agent fluid so that the line to the Formula (6) can be draw out.

In Fig.10, the circles or triangles mean the experimental data of the magnet field gradient $B B^{\prime}$ according to the different particle density $\rho_{\mathrm{p}}$ and the line is obtained from the magneto-Archimedes formula based on the fixed magneto-Archimedes agent solution. Fig.10 shows that the formula line is quite close to the experimental data. Fig.10 confirms that the Formula (5) and (7) are reasonable and can be used in future experiment and for design purpose. The similar results about $3 \mathrm{M}$ manganese (II) chloride and $4 \mathrm{M}$ manganese (II) chloride which are Fig.11 and Fig.12 are showed below.

\subsection{The effect of the shape of the container}

Fig.3 illustrates that streams of mineral particles with small size fraction were repulsed to the wall of the container when they were dropped into manganese (II) chloride solution. To confirm the phenomenon, the shape of the container was changed. Fig.13 shows the influence of the geometry factor to the repulse of the particles to container wall.

It is confirmed again that the particles in magnet field got an outward centrifugal force so that can be repulsed to the wall of the container. At the same time, the particles with different density and magnetic volume susceptibility were levitated at different positions.

\subsection{The cryogenic liquid acts as a magneto-Archimedes agent solution}

Fig.14 shows the levitation positions of different pure mineral particles in $100 \%$ liquid oxygen. Compared with $4 \mathrm{M}$ manganese (II) chloride solution, the levitation heights of the minerals in $100 \%$ liquid oxygen were much higher. Even the hematite and chalcopyrite particles which have relatively stronger paramagnetic properties can be levitated. It can be confirmed again that by increasing the paramagnetic properties of the surrounding magneto-Archimedes agent solution, paramagnetic objects in it can be levitated. 
Fig. 15 shows the levitation positions of different pure mineral particles in the mixture of $25 \%$ liquid oxygen and $75 \%$ liquid nitrogen. The levitation heights of the same minerals in mixture of $25 \%$ liquid oxygen and $75 \%$ liquid nitrogen were lower than in $100 \%$ liquid oxygen but still higher than in $4 \mathrm{M}$ manganese (II) chloride solution. But the increase of the levitation heights of the particles from $B_{c}=10 T$ to $B_{c}=16 T$ in $4 \mathrm{M}$ manganese (II) chloride solution is more significant than in the mixture fluid.

Liquid oxygen is paramagnetic, liquid nitrogen does not have any paramagnetic property, perhaps the liquid oxygen molecule moved following the magnetic strength line, accumulated and formed an enriched zone, so when $14<B c<15$, glass or bronze particles levitation positions appeared a sharp increase.

But we still can conclude that for most particles, the increase in Bc cannot help the separation.

\section{Conclusions}

In this paper, mineral particles were levitated in the magneto agent manganese (II) chloride. And the mineral particles with different density and magnetic volume susceptibility were levitated at different heights similar to the results obtained previously by others $[2,20,7,6]$.

It was confirmed that the difference of the particle densities is more significant, the more effective the separation will be. It is concluded the particles levitation heights were higher when the magnetic field strength is stronger. Besides that, the formula line obtained from previous researchers and experimental data were compared as well. It proves that the Formula (5) can be used for later check and design purpose for the object levitation in the magneto-Archimedes agent solution.

Compared with other chemical solutions, the manganese (II) chloride solution is stable, cheap and has a stronger paramagnetic property. However, this solution is toxic, so a new kind of cryogenic liquid which is composed of liquid oxygen was investigated. It is found out that the pure liquid oxygen has a stronger paramagnetic property than the mixture of liquid oxygen and liquid nitrogen. The levitation positions of particles were higher in pure liquid oxygen than the mixture fluid at corresponding magnet bore centre field strength. 
Compared with the solution $4 \mathrm{M}$ manganese (II) chloride, the levitation positions for the same particles are much higher in pure liquid oxygen. The levitation positions are somewhat lower in $4 \mathrm{M}$ manganese (II) chloride than the mixture of liquid oxygen and liquid nitrogen. The cryogenic liquid which is similar with the composition of liquid air, can effectively separate most kinds of pure mineral particles. It has low viscosity, strong paramagnetic property, and is atmosphere friendly. As the cryogenic liquid was easy to be vaporized so the particles separated from the liquid were very dry. In conclusion, the liquid air provides a potential possibility to act as the magneto-Archimedes agent in future.

\section{Acknowledgements}

This work was funded as part of the UK Engineering and Physical Sciences Research Council Basic Technology Programme: Magnetic Levitation Technology for Mineral Separation, Nanomaterials, and Biosystems for Space Exploration (GR/S83005/01)

\section{Reference:}

[1]Svoboda, J. (2004), 'Magnetic Techniques for the Treatment of Materials', Kluwer Academic Publisher

[2] Beaugnon, E. and Tournier, R. (1991), 'Levitation of water and organic substances in high static magnetic fields", Nature, Vol. 349, pp. 470

[3] Braunbeck, W. (1939), 'Magnetic levitation of graphite', Z. Physics, Vol. 112, pp. 735.

[4] Chiang, T. C. (2004), 'Magnetic Levitation at Room Temperature'

[5] Dunne, P. A., Hilton, J. and Coey, J. M. D. (2007), 'Levitation in paramagnetic liquids', Journal of Magnetism and Magnetic Materials, Vol. 316, pp. 273-276

[6] Hirota, N., Kurashige, M., Iwasaka, M., Ikehata, M., Uetake, H., Takayama, T., Nakamura, H., Ikezoe, Y., Ueno, S., and Kitazawa, K. (2004), 'Magneto-Archimedes separation and its application to the separation of biological materials', Physica B, Vol. 346-347, pp. 267-271

[7] Ikezoe, Y., Kaihatsu, T., Sakae, S., Uetake, H., Hirota, N. and Kitazawa, K. (2002),

'Separation of feeble magnetic particles with magneto-Archimedes levitation', Energy

Conversion and Management, Vol. 43, pp. 417-425 
[8] Rosensweig, R. E. (1966), 'Buoyancy and stable levitation of a magnetic body immersed in a magnetizable fluid', Nature, Vol. 210 (5036), pp. 613-614.

[9] Rosensweig, R. E. (1966), 'Fluidmagnetic Buoyancy', AlAA Journal, Vol. 4 (10), pp. 1751-1758.

[10] Rosenweig, R.E.(1997), 'Ferrohydrodynamics', Dover publications, New York. ISBN $0-486-67834-2$.

[11] Catherall, T. A., Eaves, L., King, J. P. and Booth, R. S. (2003), 'Floating gold in cryogenic Oxygen', Nature, Vol. 422, April, pp.579

[12] Catherall, T. A., Lopez-Alcaraz, P., Benedict, K. A., King, J. P. and Eaves, L. (2005), ‘Cryogenically enhanced magneto-Archimedes levitation', New J. Phys. Vol. 7, pp. 118

[13] Lopez-Alcaraz, P., Catherall, A. T., Hill, R. J. A., Leaper, M. C., Michael R. Swift, M. R. and King, P. J. (2007), 'Magneto-vibratory separation of glass and bronze granular mixtures immersed in a paramagnetic liquid', The European Physical Journal E: Soft Matter and Biological Physics, Vol.24, pp. 145-156.

[14] Andres, U. (1975), 'Magnetohydrodynamic and Magnetohydrostatic Separation - A new prospect for mineral separation in the magnetic field', Mineral Science Engineering, Vol. 7, No.2, April, pp. 99-109

[15] Suwa, M. and Watarai, H. (2002), 'Magnetophoretic Velocimetry of Manganese(II) in a Single Microdroplet in a Flow System under a High Gradient Magnetic Field Generated with a Superconducting Magnet', Anal. Chem. Vol. 74, pp. 5027-5032

[16] Rnstein, Landolt-B. (1986), 'Numerical Data and Functional Relationships in Science and Technology', New Series, II/16, Diamagnetic Susceptibility, Springer-Verlag, Heidelberg [17] Arrighini, G. P., Maestro, M. and Moccia, R. (1968), 'Magnetic Properties of Polyatomic Molecules: Magnetic Susceptibility of $\mathrm{H}_{2} \mathrm{O}, \mathrm{NH}_{3}, \mathrm{CH}_{4}, \mathrm{H}_{2} \mathrm{O}_{2}^{\prime}$, J. Chem. Phys. Vol. 49, pp. 882-889.

[18] Bennett, L. H., Page, C. H. and Swartzendruber, L. J. (1978), 'Comments on units in magnetism', Journal of Research of the National Bureau of Standards, Vol. 83 (1), pp. 9-12 [19] Hunt, C. P., Moskowitz, B. M. and Banerjee, S. K. (1995), 'Magnetic Properties of Rocks and Minerals', American Geophysical Union. 
[20] Berry, M. V. and Geim, A. K. (1997), 'Of flying frogs and levitrons', The European Physical Society, Vol. 18, pp. 307-313

\section{NOMENCLATURE}

\section{Variables}

\begin{tabular}{|c|c|c|}
\hline$B$ & the magnetic induction field strength & $(\mathrm{T})$ \\
\hline$B^{\prime}$ & vertical direction magnetic field gradient & $(T / m)$ \\
\hline$C_{\text {salt }}$ & mass percentage of salt in the total mass & $(-)$ \\
\hline$g$ & the acceleration of gravity & $\left(\mathrm{m} / \mathrm{s}^{2}\right)$ \\
\hline$k$ & volume magnetic susceptibility & $(-)$ \\
\hline$k_{1}\left(\right.$ or $\left.k_{p}\right)$ & mass susceptibility of the levitating substances & $(-)$ \\
\hline$k_{2}\left(\right.$ or $\left.k_{l}\right)$ & mass susceptibility of the medium gas (or liquid) & $(-)$ \\
\hline$m$ & the mass of the particle & $(\mathrm{kg})$ \\
\hline$V$ & particle volume & $\left(m^{3}\right)$ \\
\hline$x$ & mass magnetic susceptibility & $\left(\mathrm{m}^{3} / \mathrm{kg}\right)$ \\
\hline$\mu_{0}$ & the permeability of free space & $(H / m)$ \\
\hline$\rho$ & mass density & $\left(\mathrm{kg} / \mathrm{m}^{3}\right)$ \\
\hline$\rho_{1}$ & the density of the levitating substances & $\left(\mathrm{kg} / \mathrm{m}^{3}\right)$ \\
\hline$\rho_{a}$ & the apparent density of liquid & $\left(\mathrm{kg} / \mathrm{m}^{3}\right)$ \\
\hline$\rho_{2} \quad\left(\right.$ or $\left.\rho_{l}\right)$ & density of medium gas (or liquid) around it & $\left(\mathrm{kg} / \mathrm{m}^{3}\right)$ \\
\hline$\rho_{L}$ & actual density of liquid & $\left(\mathrm{kg} / \mathrm{m}^{3}\right)$ \\
\hline \multicolumn{3}{|l|}{$d B$} \\
\hline$\overline{d z}$ & vertical direction magnetic field gradient & $(\mathrm{T} / \mathrm{m})$ \\
\hline
\end{tabular}


Table.1: The density and volume magnetic susceptibility of different concentrations manganese (II) chloride solution

\begin{tabular}{|c|c|c|c|}
\hline \multicolumn{2}{|r|}{ Solution } & Density $\left(\mathrm{kg} \cdot \mathrm{m}^{-3}\right)$ & $k \times 10^{-6}$ \\
\hline $2 \mathrm{~N}$ & Manganese(II) Chloride & 1227 & 345 \\
\hline $3 \mathrm{~N}$ & Manganese(II) Chloride & 1301 & 502 \\
\hline $4 \mathrm{~N}$ & Manganese(II) Chloride & 1395 & 660 \\
\hline
\end{tabular}

Table.2: The density, volume susceptibility and mass susceptibility of different materials (Andres, 1975; Hunt et al., 1995; Gregory Bottley and Lloyd)

\begin{tabular}{|c|c|c|c|c|}
\hline Materials & $\begin{array}{c}\text { Chemical } \\
\text { Form }\end{array}$ & $\begin{array}{c}\text { Density } \\
\left(\mathrm{kg} . \mathrm{m}^{-3}\right)\end{array}$ & $k_{\mathrm{p}} \times 10^{-6}$ & $x_{\mathrm{p}} \times 10^{-9}\left(\mathrm{~m}^{3} \cdot \mathrm{kg}^{-1}\right)$ \\
\hline Pyrite & $\mathrm{FeS}_{2}$ & 4654 & 314 & 67.5 \\
\hline Quartz & $\mathrm{SiO}_{2}$ & 2650 & -15.1 & -5.7 \\
\hline Calcite & $\mathrm{CaCO}_{3}$ & 2710 & -10.3 & -3.8 \\
\hline Chalcopyrite & $\mathrm{CuFeS}_{2}$ & 4250 & 6783 & 1596 \\
\hline Dolomite & $\mathrm{CaMg}_{2}\left(\mathrm{CO}_{3}\right)_{2}$ & 2974 & 45 & 15.1 \\
\hline Galena & $\mathrm{PbS}$ & 7230 & -31.8 & -4.4 \\
\hline Goethite & $\mathrm{HFeO}_{2}$ & 4300 & $1075-1634$ & $250-380$ \\
\hline Hematite & $\mathrm{Fe}_{2} \mathrm{O}_{3}$ & 5245 & $2622-19931$ & $500-3800$ \\
\hline Rutile & $\mathrm{TiO}_{2}$ & 4200 & $50-210$ & $12-50$ \\
\hline Sphalerite & $\mathrm{ZnS}$ & 4000 & -13.2 & -3.3 \\
\hline Wolframite & $(\mathrm{MnFe}) \mathrm{WO}_{4}$ & 7000 & $2660-8400$ & $380-1200$ \\
\hline Glass/Sand & $(-)$ & 2650 & $(-)$ & $(-)$ \\
\hline
\end{tabular}




\section{List of Figures}

Fig. 1: MCV magnet system used in experiments

Fig. 2: The vessel in the magnet bore centre hole

Fig. 3: Schematic of ring formation of pyrite particles levitating in a manganese (II) chloride solution

Fig. 4: The photograph of quartz and pyrite particles levitated in $4 \mathrm{M} \mathrm{MnCl} 2$ solution in a column container under $\mathrm{Bc}=16.5 \mathrm{~T}$

Fig. 5: The different levitation heights at different field strengths in $4 \mathrm{M}$ manganese (II) chloride solution

Fig. 6: Floating heights of minerals above the magnet bore in 3M manganese(II) chloride at various values of $\mathrm{BC}$

Fig.7: Floating heights of minerals above the magnet bore in $2 \mathrm{M}$ manganese(II) chloride at various values of $\mathrm{Bc}$

Fig. 8: The levitation positions of the particles with different density in $4 \mathrm{M}$ manganese (II) chloride solution at $\mathrm{Bc}=16 \mathrm{~T}$

Fig.9: The levitation positions of quartz particles at different Bc in $4 \mathrm{M}$ manganese (II) chloride solution

Fig.10: The comparison of the line obtained from independently-measured values of $\rho$ and $\kappa$ and plot of experimental BB' against particle density for 2M manganese (II) chloride

Fig.11: Plot of experimental BB' against particle density for 3M manganese (II) chloride, compared with the line obtained from independently-measured values of $\rho$ and $\kappa$

Fig.12: Plot of experimental BB' against particle density for 4M manganese (II) chloride, compared with the line obtained from independently-measured values of $\rho$ and $\kappa$

Fig. 13: The levitation of glass and pyrite particles in elliptical container in $4 \mathrm{M} \mathrm{MnCl} 2$ solution

Fig. 14: The different pure mineral particles levitated at different positions in $100 \%$ liquid oxygen at different $\mathrm{Bc}$

Fig. 15: The different pure mineral particles levitated at different positions in the mixture of $25 \%$ liquid oxygen and $75 \%$ liquid nitrogen in volume at different $\mathrm{Bc}$

Fig. 16: The schematic force on the solution in the tube

All the colour figures are intended for colour reproduction on the web and black-and-white in print. 


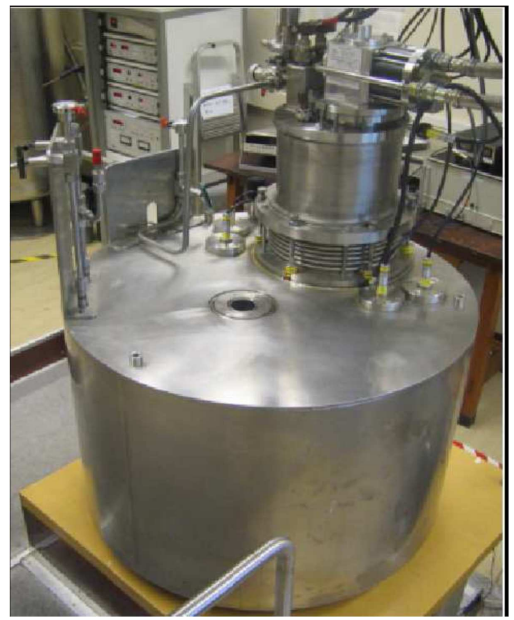

a. MCV magnet system

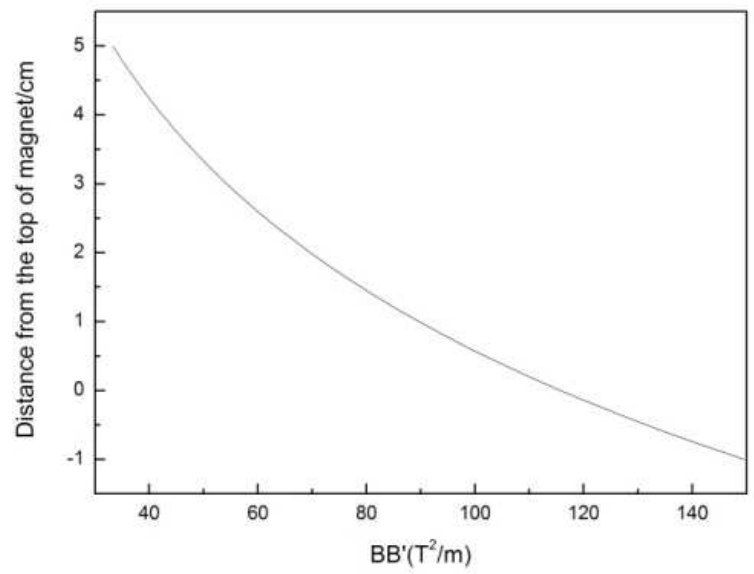

b. $\mathrm{BB}^{\prime}$ Vs distance from the top of magnet

Fig. 1: MCV magnet system used in experiments

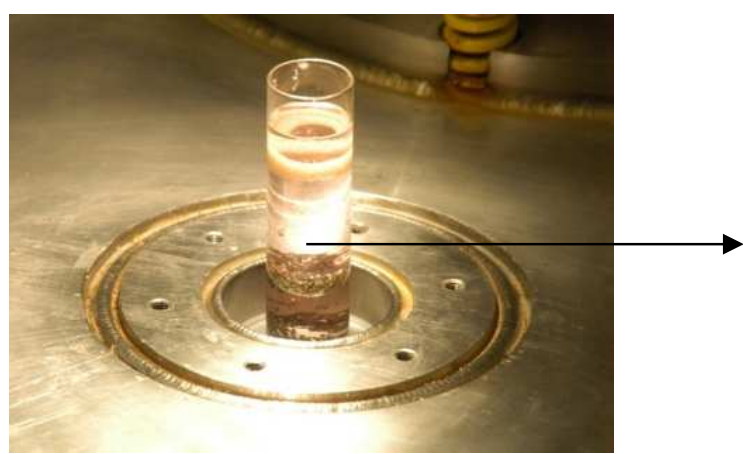

The vessel on the platform in the magnet bore hole

Fig. 2: The vessel in the magnet bore centre hole

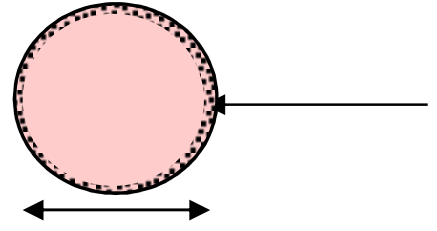

$23 \mathrm{~mm}$ plan view - particles forced to edge of container almost a monoparticle layer

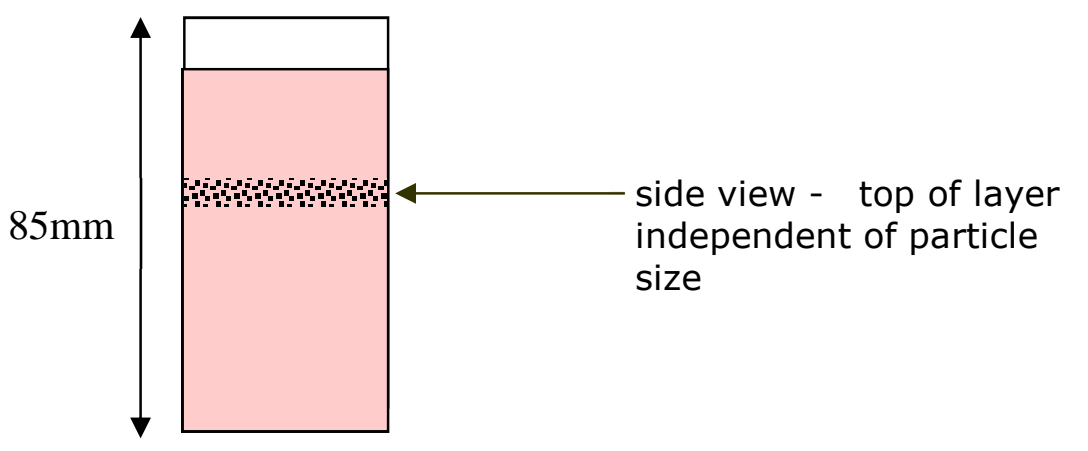

Fig. 3: Schematic of ring formation of pyrite particles levitating in a manganese (II) chloride solution 


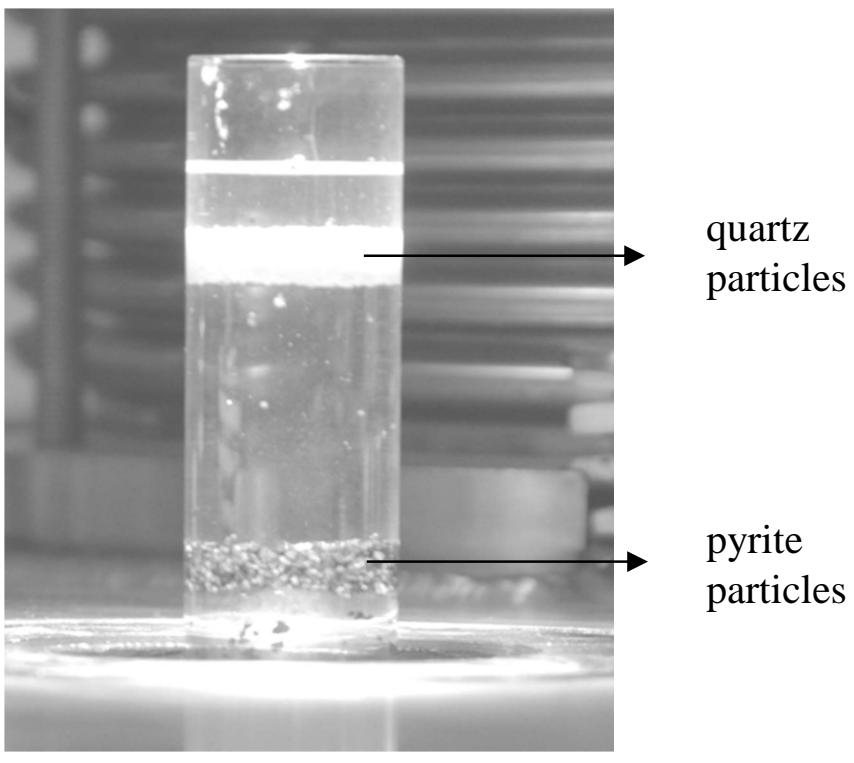

Fig.4: The photograph of quartz and pyrite particles levitated in $4 \mathrm{M} \mathrm{MnCl}_{2}$ solution in a column container under $\mathrm{B}_{\mathrm{c}}=16.5 \mathrm{~T}$

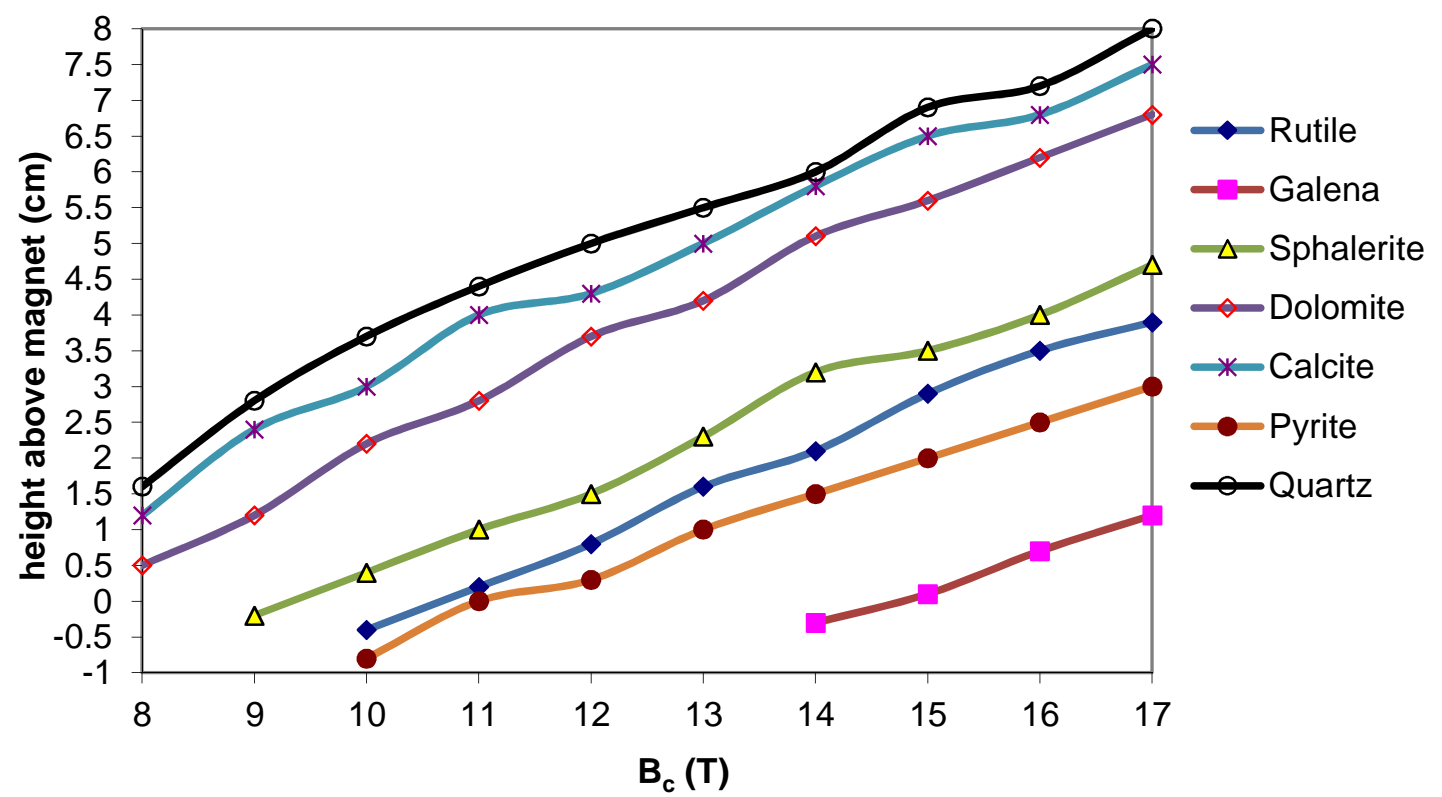

Fig. 5: The different levitation heights at different field strengths in $4 \mathrm{M}$ manganese (II) chloride solution 


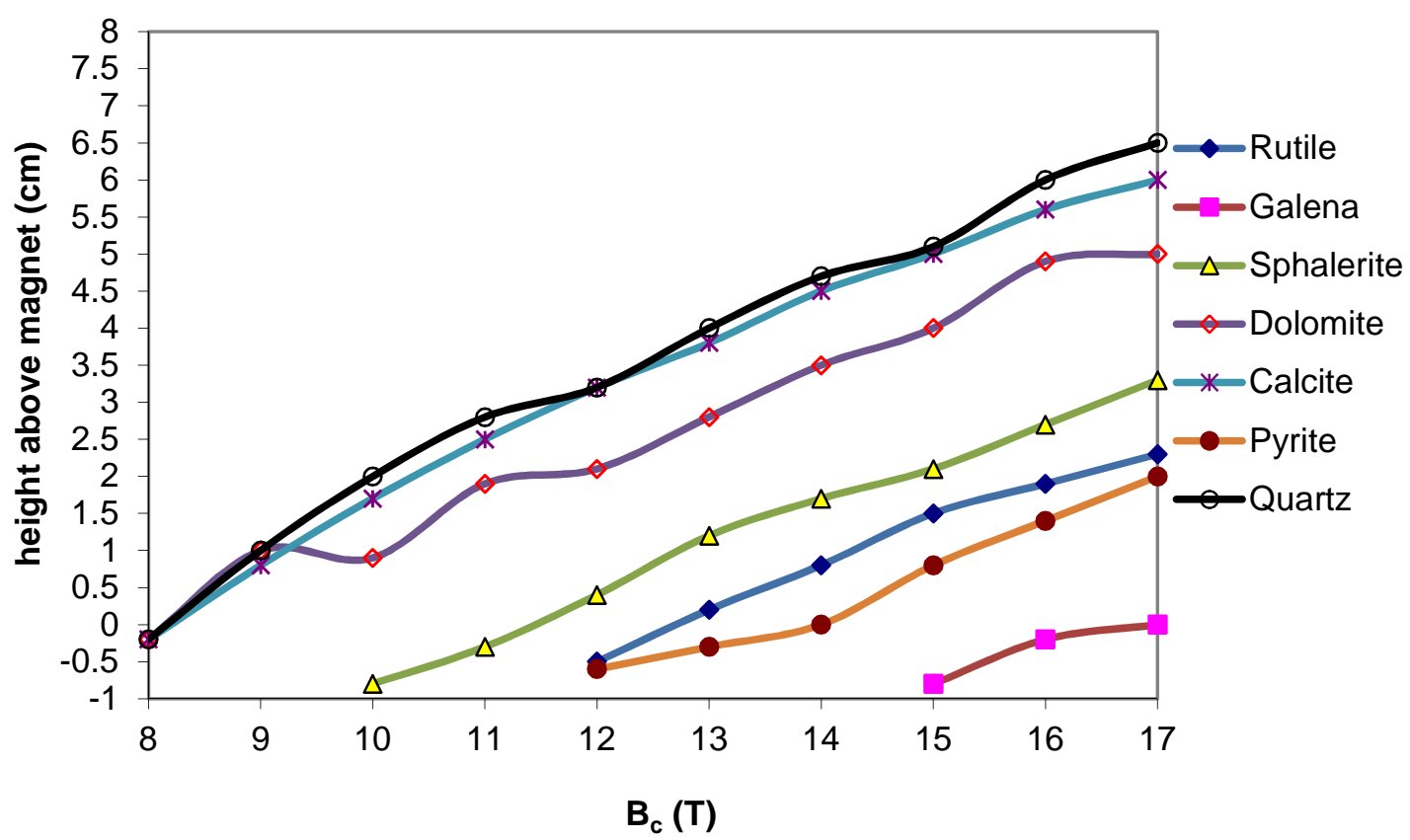

Fig. 6: Floating heights of minerals above the magnet bore in 3M manganese(II) chloride at various values of $B_{c}$

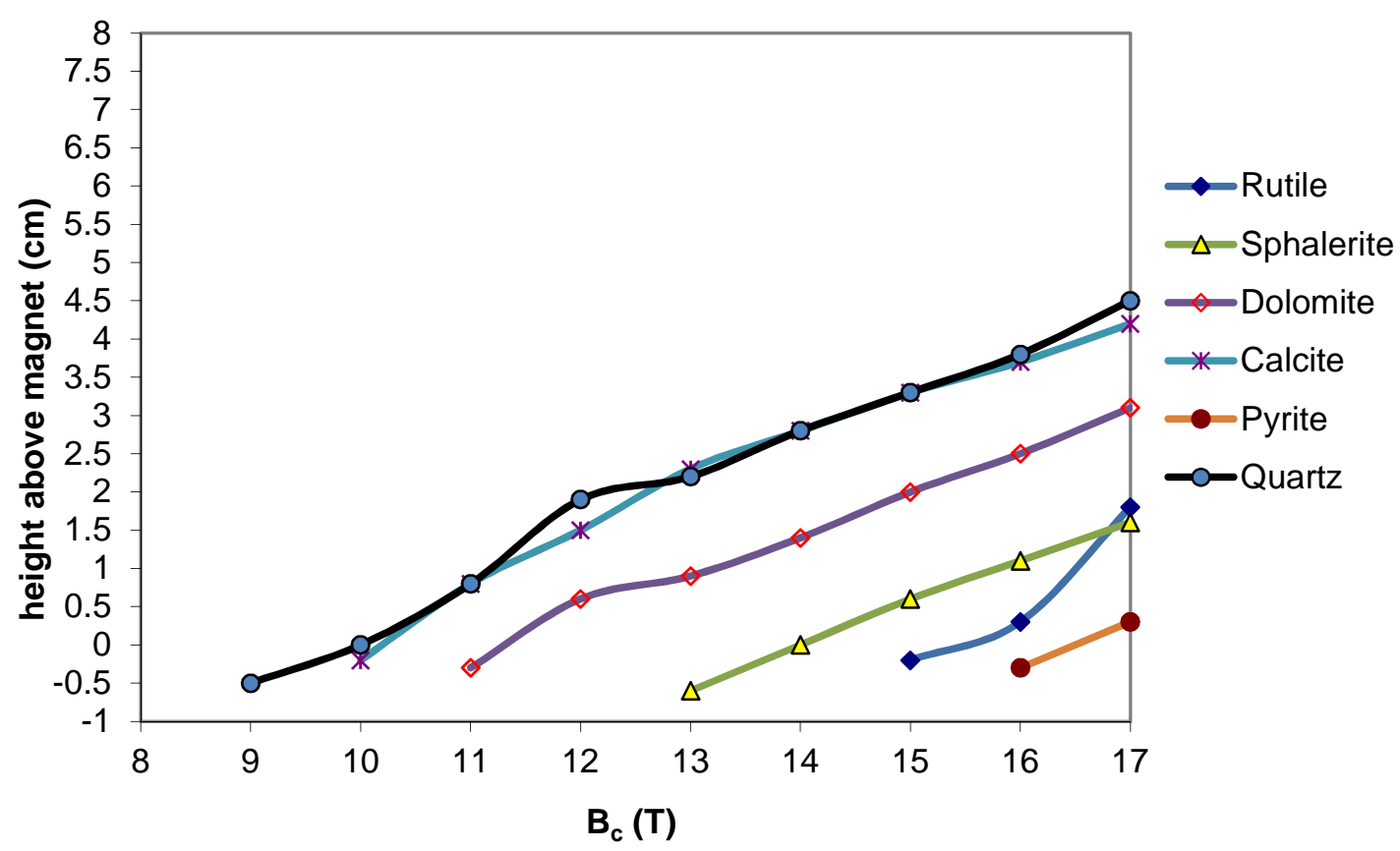

Fig. 7: Floating heights of minerals above the magnet bore in $2 \mathrm{M}$ manganese(II) chloride at various values of $B_{c}$ 


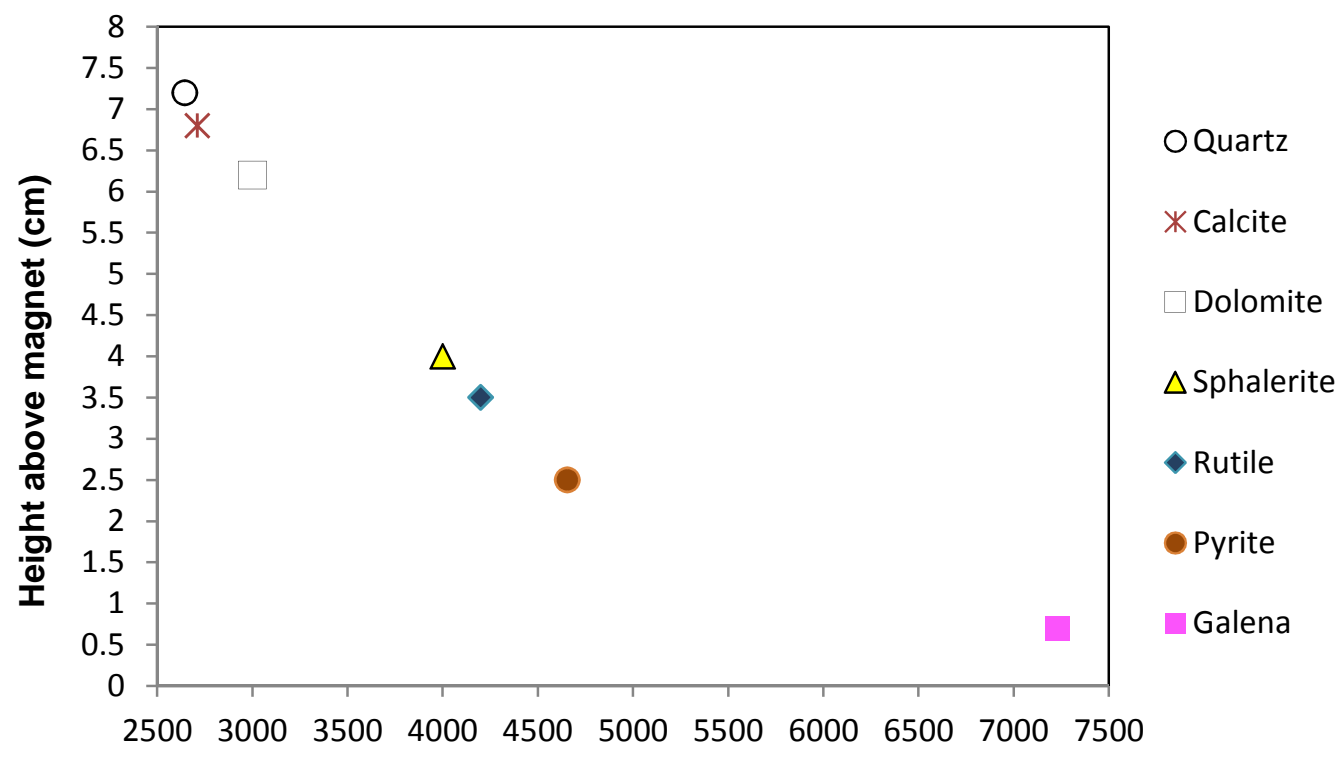

Particle Density $\left(\mathrm{kg} \cdot \mathrm{m}^{-3}\right)$

Fig. 8: The levitation positions of the particles with different density in $4 \mathrm{M}$ manganese (II) chloride solution at $B_{c}=16 T$

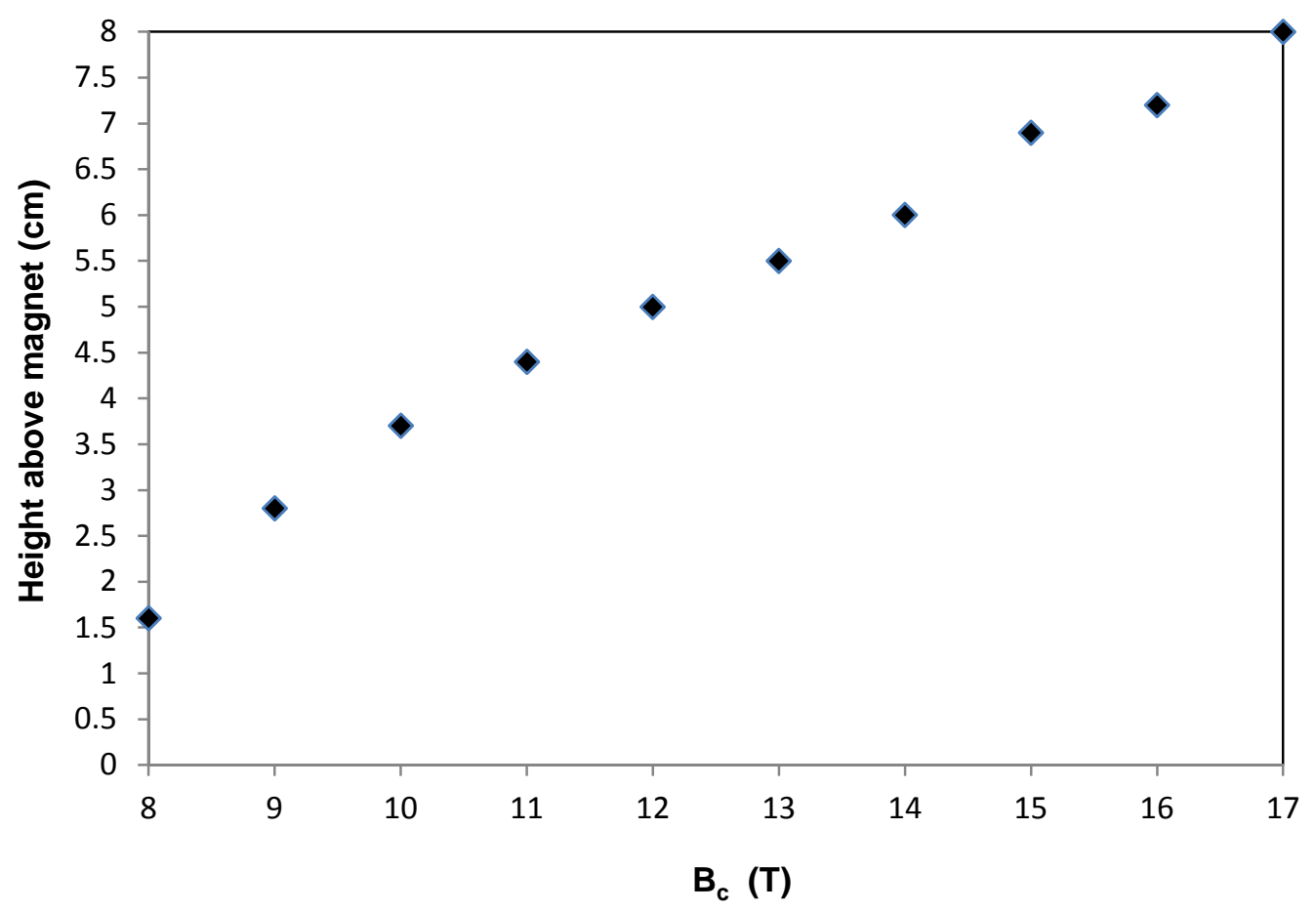

Fig. 9: The levitation positions of quartz particles at different $B_{c}$ in $4 \mathrm{M}$ manganese (II) chloride solution 


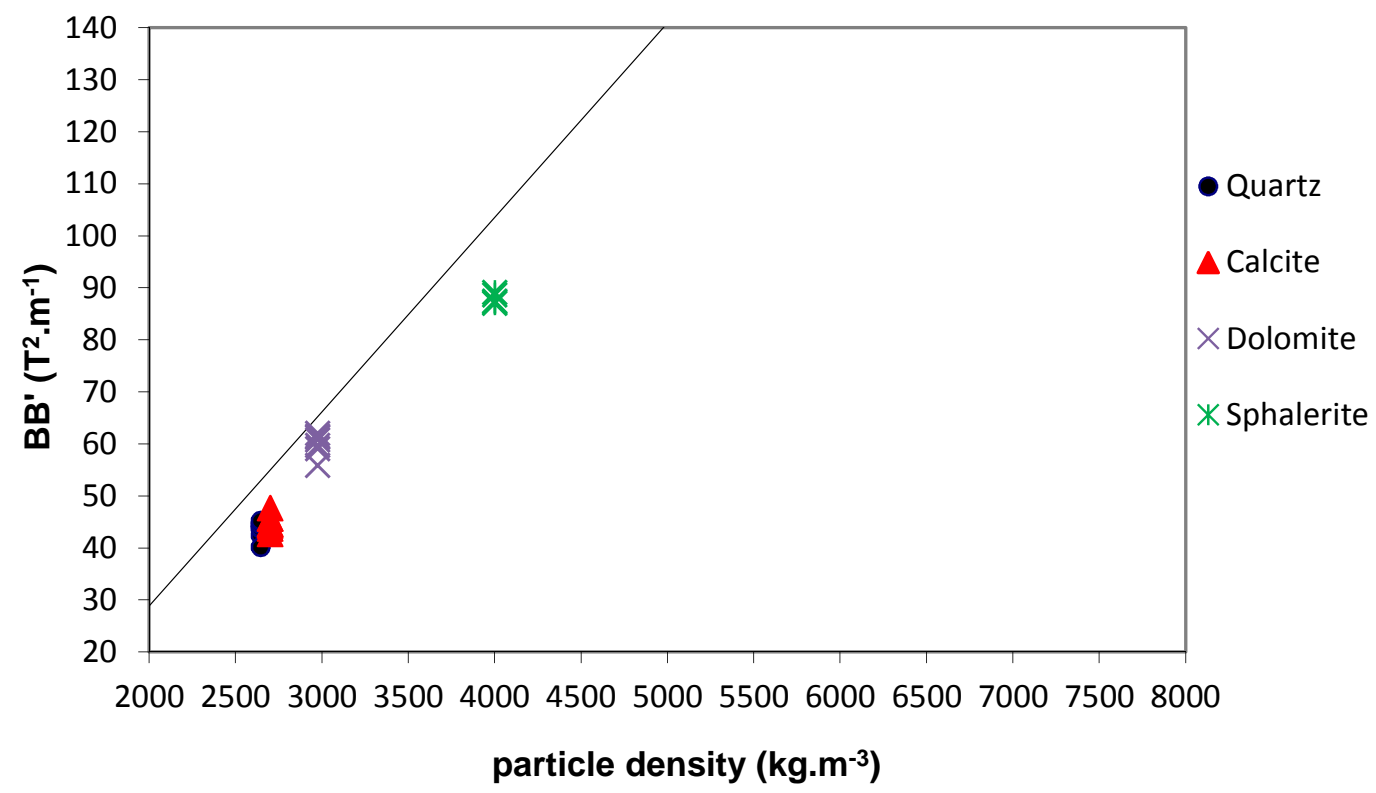

Fig. 10: The comparison of the line obtained from independently-measured values of $\rho$ and $\kappa$ and plot of experimental BB' against particle density for 2M manganese (II) chloride

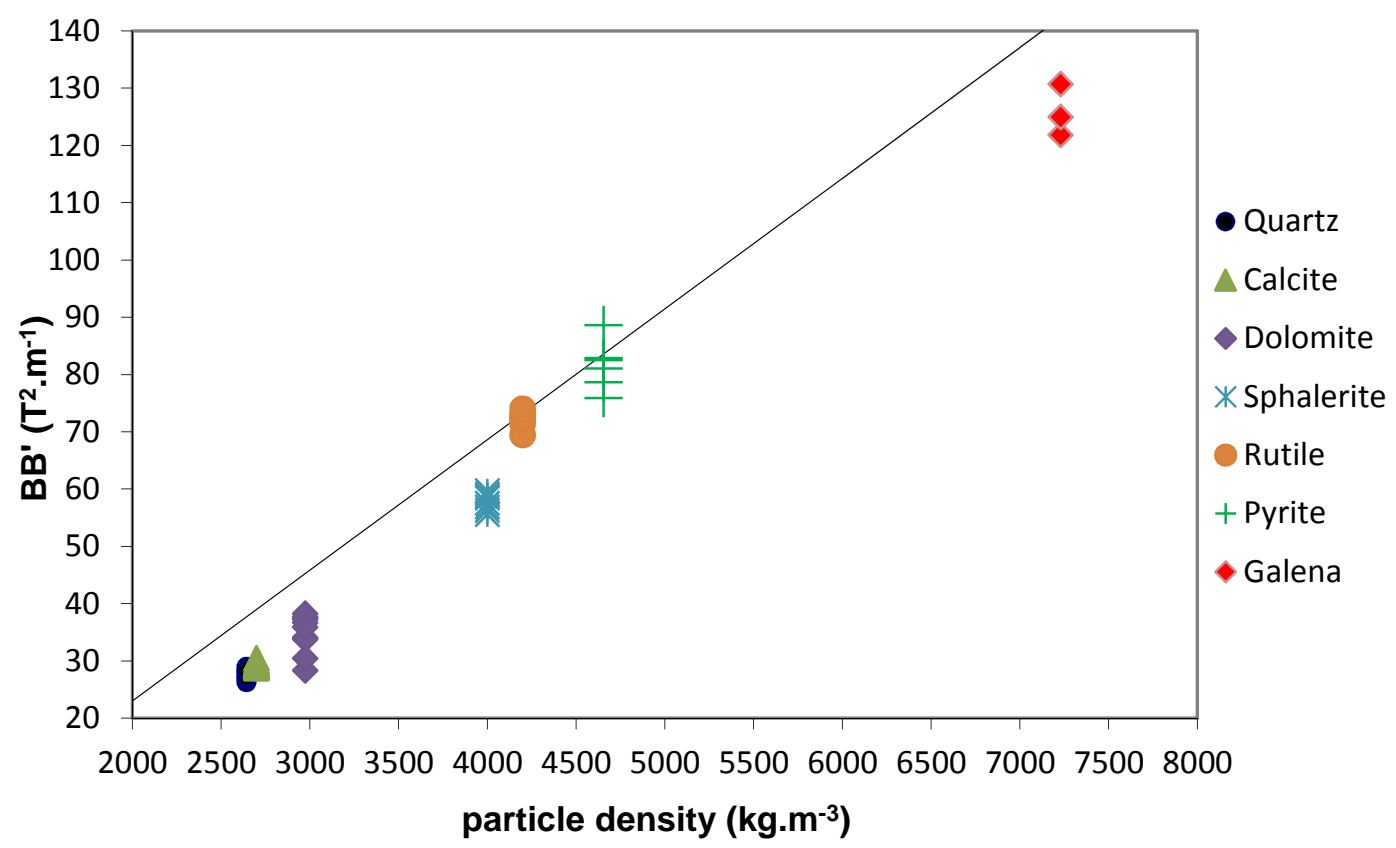

Fig. 11: Plot of experimental BB' against particle density for $3 \mathrm{M}$ manganese (II) chloride, compared with the line obtained from independently-measured values of $\rho$ and $\kappa$ 


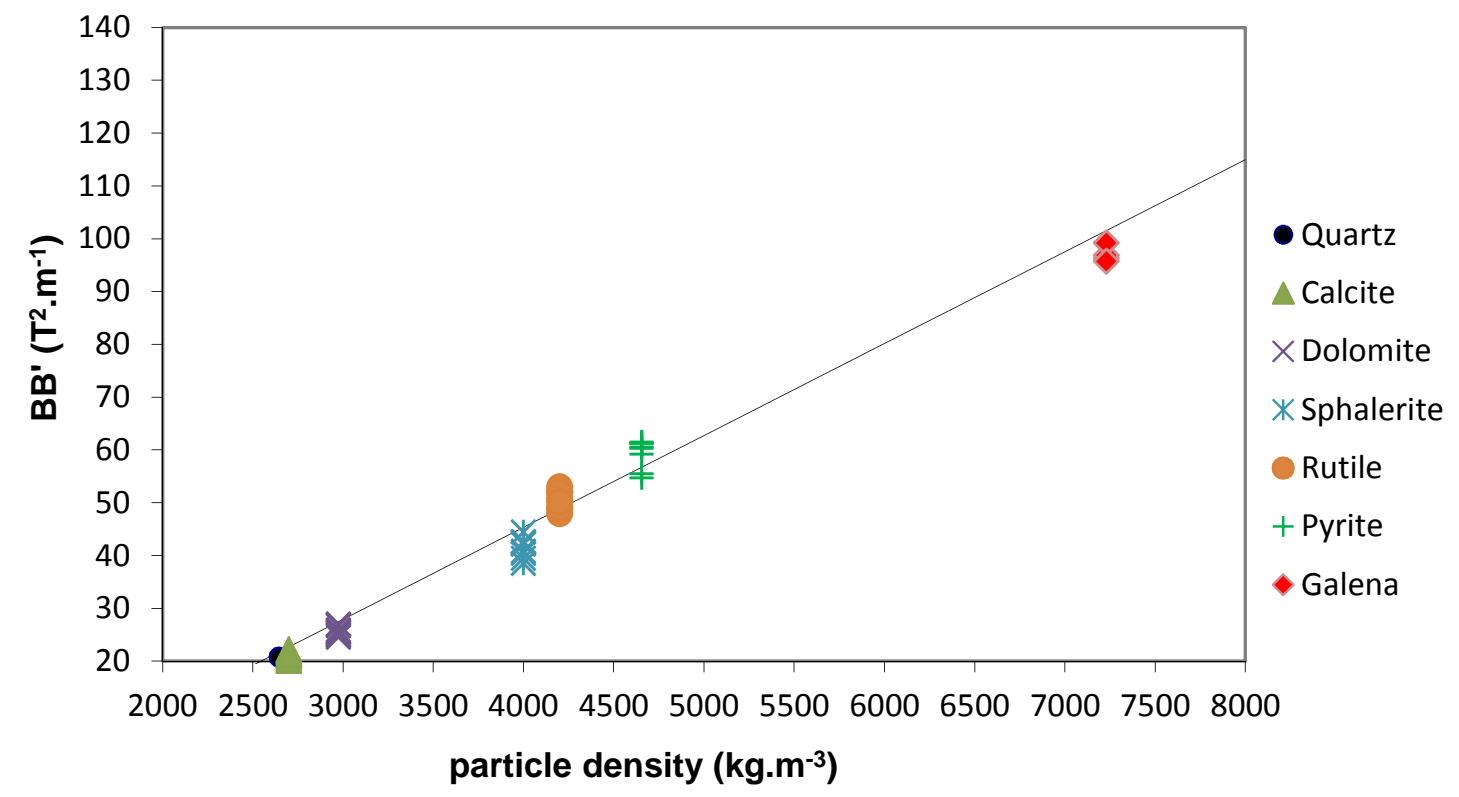

Fig. 12: Plot of experimental BB' against particle density for $4 \mathrm{M}$ manganese (II) chloride, compared with the line obtained from independently-measured values of $\rho$ and $\kappa$

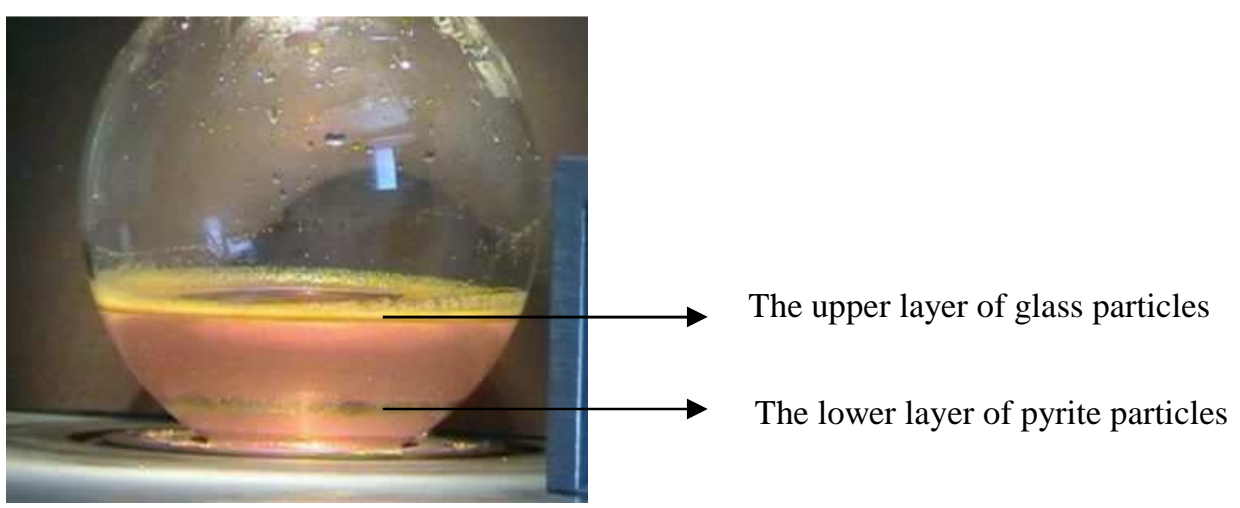

Fig. 13: The levitation of glass and pyrite particles in elliptical container in $4 \mathrm{M} \mathrm{MnCl}_{2}$ solution 


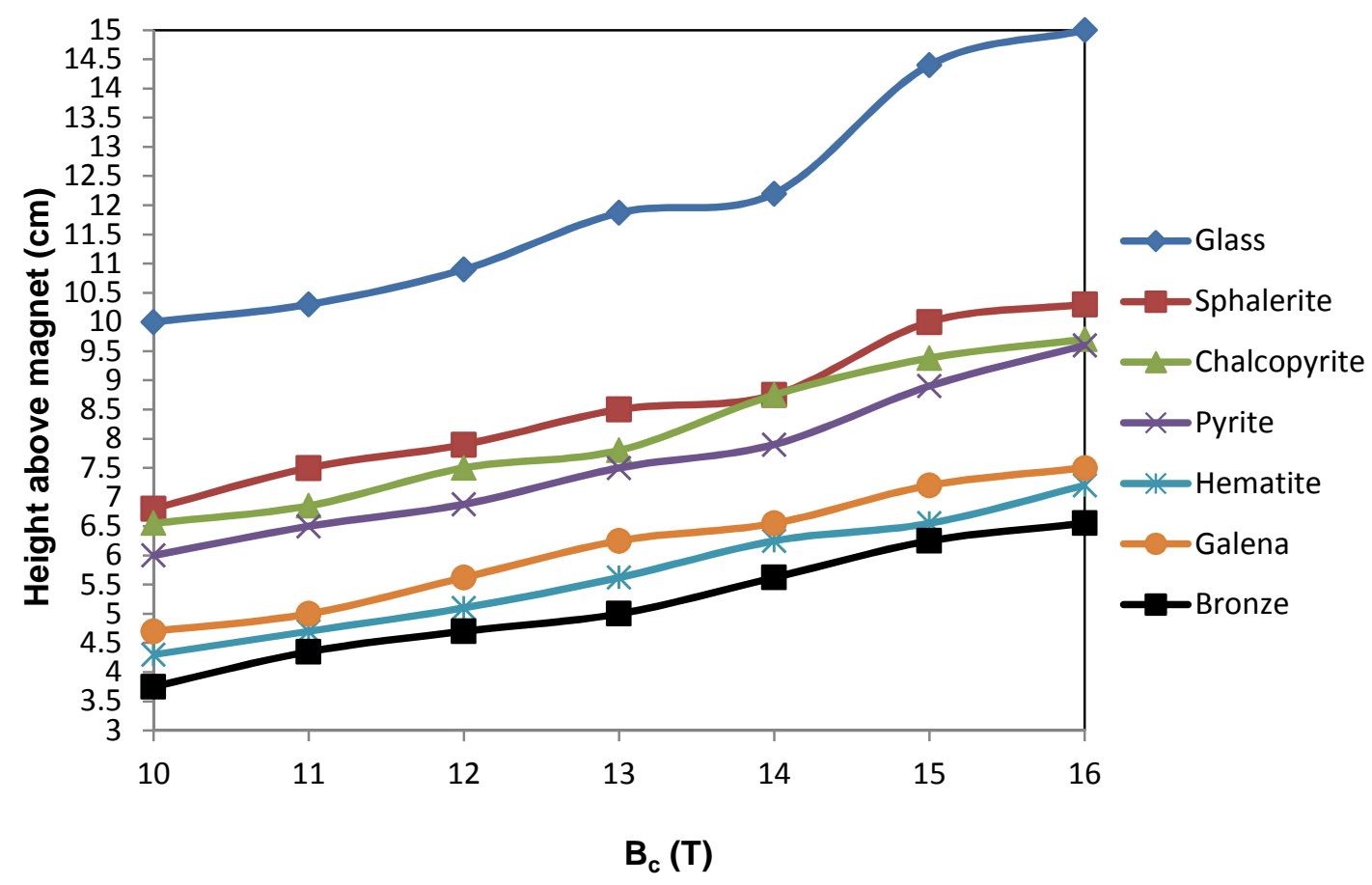

Fig.14: The different pure mineral particles levitated at different positions in $100 \%$ liquid oxygen at different $B_{c}$

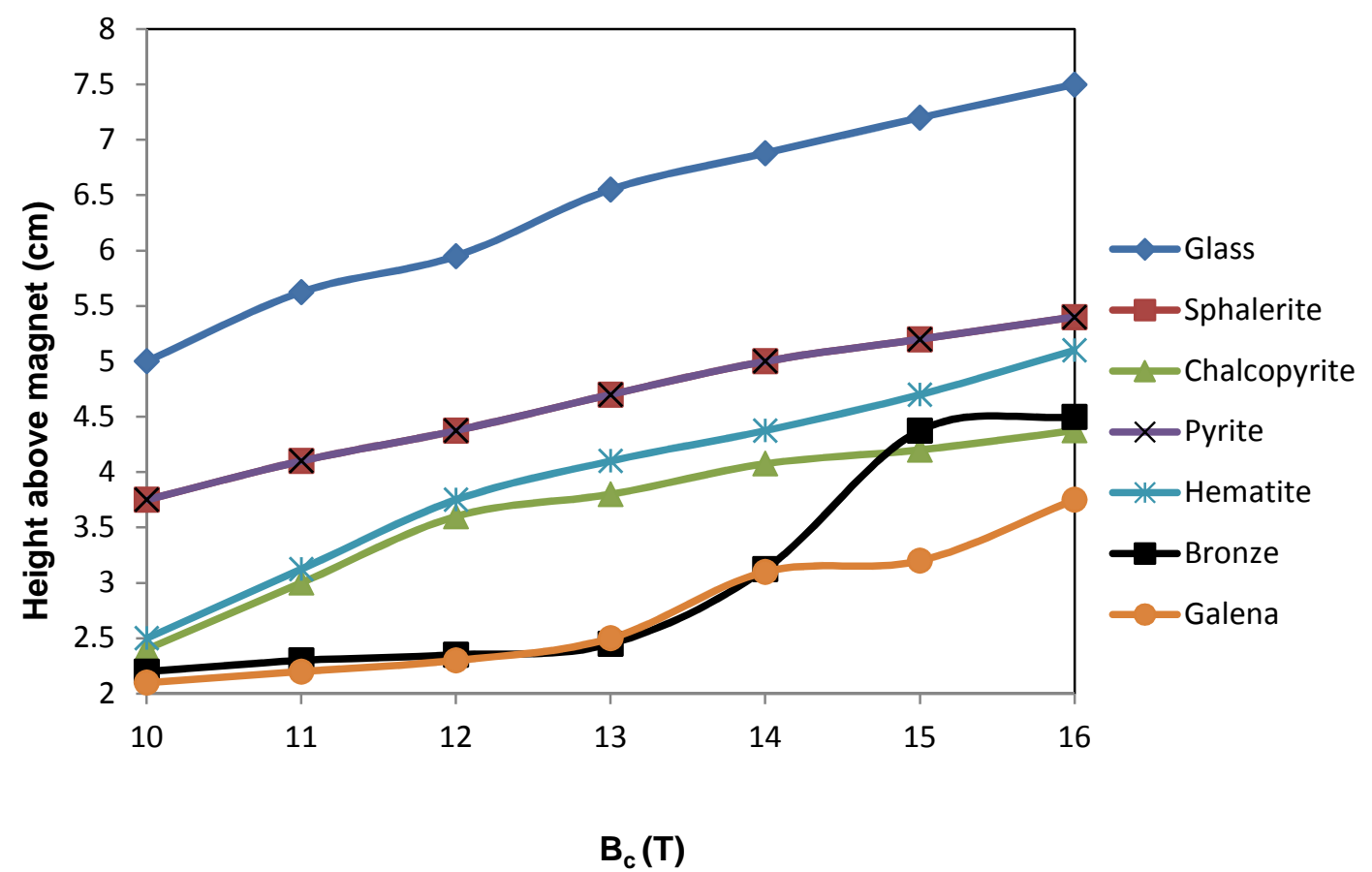

Fig. 15: The different pure mineral particles levitated at different positions in the mixture of $25 \%$ liquid oxygen and $75 \%$ liquid nitrogen in volume at different $B_{c}$ 